\title{
CUIDADO DE CRIANÇAS E ADOLESCENTES COM NECESSIDADES ESPECIAIS DE SAÚDE: ESTUDO DE REVISÃO NARRATIVA ${ }^{1}$
}

\author{
CARE OF CHILDREN AND TEENAGERS WITH SPECIAL HEALTH NEEDS: \\ A NARRATIVE REVIEW STUDY
}

\author{
Andressa da Silveira ${ }^{2}$, Mariana Padilha Werle ${ }^{3}$, \\ Keity Laís Siepmann Soccol ${ }^{4}$ e Zaira Letícia Tisott ${ }^{5}$
}

\section{RESUMO}

O objetivo do presente estudo foi conhecer a produção do conhecimento acerca do cuidado às crianças e/ou adolescentes com necessidades especiais de saúde no Brasil. Trata-se de uma revisão bibliográfica, do tipo narrativa realizada entre os meses de novembro e dezembro de 2020, nas bases de dados Scientific Electronic Library Online e Literatura Latino-Americana do Caribe em Ciências da Saúde. Foram inclusas as produções dos últimos cinco anos, produzidos no Brasil, com texto disponível nos idiomas português, espanhol ou inglês de forma gratuita. Selecionou-se para esse estudo nove artigos científicos, os quais abordam temáticas relacionadas à sobrecarga dos familiares cuidadores de crianças e adolescentes com necessidades especiais de saúde, destacam-se o cuidado familiar e materno, e ainda o autocuidado dessa população. O cuidado exercido as crianças e adolescentes com necessidades especiais de saúde podem gerar sobrecarga às famílias, principalmente à mãe. Por fim, o estudo denota sobre a necessidade de uma rede de apoio social para a construção desse cuidado aliado a rede de serviços de saúde. Os profissionais de saúde são fundamentais no suporte aos familiares dessas crianças e adolescentes.

Palavras-chave: Saúde da criança, Saúde do adolescente, Pessoas com deficiência, Revisão.

\section{ABSTRACT}

The aim of this study was to learn about the production of knowledge about the care of children and/or adolescents with special health needs in Brazil. This is a bibliographic review, of a narrative type carried out between the months of November and December 2020, in the databases Scientific Electronic Library Online and Latin American Caribbean Literature in Health Sciences. produced in Brazil, with text available in Portuguese, Spanish or English free of charge. Nine scientific articles were selected for this study, which address themes related to the burden of family caregivers of children and adolescents with special health needs, family and maternal care, and the self-care of this population. The care provided to children and adolescents with special health needs can create an overload on families, especially on the mother. Finally, the study shows the need for a social support network to build this care combined with a health service network. Health professionals are fundamental in supporting the families of these children and adolescents.

Keywords: Child health, Adolescent health, Disabled persons, Review.

1 Estudo de Revisão Narrativa.

2 Doutora em Enfermagem. Docente do Departamento de Ciências da Saúde, Universidade Federal de Santa Maria, Campus de Palmeira das Missões. E-mail: andressa-da-silveira@ufsm.br

3 Enfermeira. Hospital de Caridade de Palmeira das Missões. E-mail: marianawe@hotmail.com

4 Doutora em Enfermagem. Docente da Universidade Franciscana. E-mail: keitylais@hotmail.com

5 Mestre em Enfermagem. Docente da Universidade Regional do Noroeste do Estado do Rio Grande do Sul. E-mail: zaira.tisott@hotmail.com 


\section{INTRODUÇÃO}

O crescente avanço tecnológico e os inúmeros achados na área da saúde possibilitaram um aumento de sobrevida na infância, contribuindo assim com a manutenção da vida de crianças com doenças graves e/ou incapacitantes, de modo que, sem esse progresso, elas não se manteriam vivas. Esse avanço tem provocado mudanças no perfil epidemiológico da infância, originando um grupo de crianças inicialmente denominadas nos Estados Unidos de Children with special health care needs (CSHCN) (MCPherson et al., 1998) e no Brasil foram denominadas como Crianças e adolescentes com necessidades especiais de saúde (CRIANES) (SILVEIRA; NEVES, 2017).

A partir deste panorama de sobrevida com condições crônicas de saúde, a população de CRIANES apresenta demandas de cuidados que podem ser classificados da seguinte forma: demanda de desenvolvimento (requerem reabilitação psicomotora e social); tecnológica (possuem algum tipo de tecnologia no corpo, por exemplo traqueostomia ou colostomia); medicamentosa (os que são farmacodependentes); habituais modificados (dependem de modificações ou adaptações para exercer as atividades do dia-a-dia, como por exemplo, auxílio para higiene, alimentação ou locomoção); mistas (associação de mais de um tipo de cuidado); e cuidados clinicamente complexos (combinação de todas as demandas anteriores e a inclusão do suporte de vida) (MCPHERSON et al., 1998; CABRAL et al., 2004).

De acordo com a Organização Mundial de Saúde (OMS), as doenças crônicas são as principais causas de mortes e incapacidades no mundo. Elas contribuem para o sofrimento dos indivíduos, das famílias e da sociedade, o que requer atenção contínua e esforços de um conjunto de equipamentos e políticas públicas (FIGUEIREDO; SOUSA; GOMES, 2016). Os dados epidemiológicos sobre as CRIANES no Brasil não existem oficialmente, o que denota a invisibilidade desta clientela em taxas oficiais. Dados mais atualizados são dos Estados Unidos da América (EUA), entre os anos de 2011-2012, e mostram que em torno de 19,8\% das crianças e adolescentes de 0-17 anos apresentam alguma necessidade especial de saúde (SILVEIRA; NEVES, 2017).

Apesar da invisibilidade de dados epidemiológicos nacionais a respeito da população de CRIANES, os dados sobre crianças e adolescentes com doenças crônicas surgem em algumas pesquisas. Entre elas, destaca-se o estudo em que as crianças entre 0 a 5 anos aproximadamente 9,1\% são acometidas por alguma doença crônica, nas de 6 a 13 anos 9,7\%, e entre as de 14 a 19 anos 11\% são acometidas por alguma doença crônica. Salienta-se, que as doenças crônicas são aquelas com duração longa ou indefinida, que apresentam prognóstico geralmente incerto e que os indivíduos podem vivenciar períodos de remissão e exacerbação sintomatológica, assim requerem um processo de cuidado contínuo sem que, necessariamente, resulte em cura (NÓBREGA et al., 2017).

A sobrevida de crianças e adolescentes com doenças crônicas, revela a necessidade de políticas de saúde voltadas a essa população. Embora a saúde da criança e do adolescente apresentem políticas públicas de saúde (BRASIL; 2011), no que se refere a saúde do adolescente especificamente, 
ainda é um desafio (SILVEIRA; NEVES, 2017). Visto que é nesta fase da vida que acontecem importantes transformações, articuladas a um envolvimento social e ao redimensionamento da sua identidade e dos novos papeis sociais que vão assumindo (BRASIL, 2017). Esses desafios podem ser ainda maiores, quando associados a outras condições de saúde, como por exemplo, aquelas que demandam cuidados especiais para a sobrevivência (SILVEIRA; NEVES, 2017).

As CRIANES necessitam de um acompanhamento na saúde que vai além dos atendimentos médicos e de enfermagem, compreende também atendimentos na área de reabilitação, apoio educacional, social e familiar. A manutenção da vida de CRIANES está relacionada a presença de cuidados técnicos especializados, individuais e personalizados que elas necessitam. Esses cuidados podem ter natureza temporária ou permanente, e de modo geral, são desenvolvidos pelo núcleo familiar, com ênfase nas mulheres da família que assumem o papel de cuidadoras primárias de CRIANES (NEVES et al., 2015).

Para a operacionalização deste processo de cuidado é fundamental a integração entre os profissionais de saúde e dos familiares/cuidadores, para que o cuidado contemple integralmente as necessidades da CRIANES e sua família (INÁCIO; PEIXOTO, 2017). Ressalta-se ainda, que o cuidado a essas crianças e adolescentes é complexo, exigindo da família a aquisição de novas habilidades e competências para o cuidado. Desta forma, o papel desempenhado pela família de pacientes pediátricos requer um compromisso entre a equipe de saúde e do núcleo familiar, para a continuidade dos cuidados de manutenção da vida (LEITE et al., 2019).

Frente ao exposto, este estudo tem por objetivo conhecer a produção do conhecimento acerca do cuidado às crianças e/ou adolescentes com necessidades especiais de saúde no Brasil.

\section{MÉTODO}

Trata-se de uma revisão bibliográfica, do tipo revisão narrativa. De acordo com Rother (2007), as revisões narrativas são publicações amplas, que possuem o propósito de descrever e discutir o desenvolvimento do estado da arte sobre um determinado tema. Essa análise possibilita a identificação de lacunas de conhecimento, atuando como subsídio para a realização de novas pesquisas, fazendo com que as revisões narrativas contribuam no debate de determinados temas, e colaborem na aquisição e atualização do conhecimento.

O estudo foi realizado entre os meses de novembro e dezembro de 2020, nas bases de dados Scientific Electronic Library Online (SCIELO) e Literatura Latino-Americana do Caribe em Ciências da Saúde (LILACS). A questão de pesquisa utilizada para este estudo foi: Qual é a produção do conhecimento nacional atual acerca do cuidado de crianças e/ou adolescentes com necessidades especiais de saúde?

Utilizou-se como critérios de inclusão produções dos últimos cinco anos, período justificado devido as amplas modificações na produção do conhecimento e por se tratar de um estudo de revisão, 
com ênfase em produções atuais no Brasil. Incluíram-se ainda, produções nos idiomas português, espanhol ou inglês de forma gratuita. Excluíram-se manuais, e-books, monografias, dissertações, teses, produções que estivessem duplicadas e aquelas que não convergiam com o objetivo proposto neste estudo.

Para a operacionalização do estudo utilizou-se os descritores "cuidado da criança" [AND] "saúde do adolescente" [AND] "brasil" por meio do formulário avançado, conforme o fluxograma a seguir:

Figura 1 - Fluxograma da estratégia de busca realizada na Scielo e Lilacs.

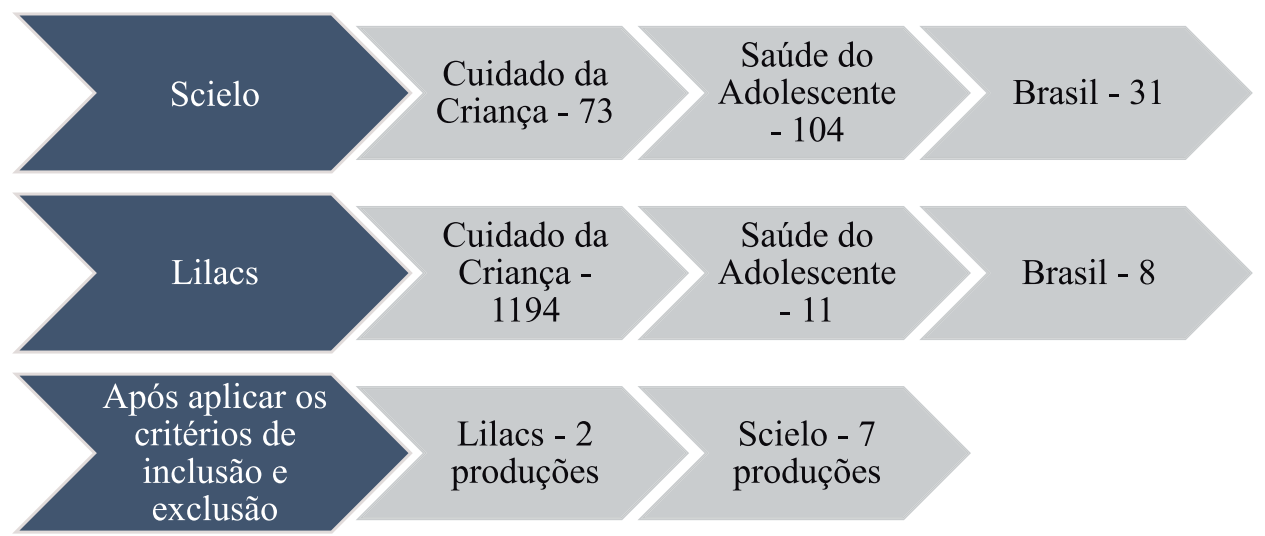

Fonte: Autoras da revisão narrativa, 2020.

A partir dos critérios de inclusão e exclusão o estudo foi constituído por nove artigos científicos que foram alocados em um quadro sinóptico, o qual possibilitou caracterizar a produção do conhecimento acerca da temática proposta.

\section{RESULTADOS E DISCUSSÃO}

Para a apresentação dos resultados das informações, os dados foram organizados por meio da construção de um quadro sinóptico que contém o código de identificação, em ordem alfabética conforme a sequência que foram citados no texto, o título, o ano de publicação, o periódico e a base de dados. Os artigos que constituíram o corpus de análise estão apresentados no Quadro 1.

Entre as temáticas apresentadas, observa-se a sobrecarga dos familiares cuidadores de CRIANES (A1), cuidado familiar (A8), cuidado materno (A4 e A9), autocuidado de crianças e adolescentes (A2, A7) e o cuidado às crianças e aos adolescentes com deficiência (A3, A5, A6).

A partir da aprovação do Estatuto da Criança e do Adolescente - ECA em 1990 (BRASIL, 1990), a criança e o adolescente tiveram seus direitos formalizados, o que implicou em alterações normativas e culturais para as políticas públicas e aos serviços de saúde que prestam atendimento à essa população. Em 2015, a Lei Brasileira de Inclusão da Pessoa com Deficiência - Estatuto da Pessoa com Deficiência garantiu às crianças e aos adolescentes com deficiência o direito à igualdade, de não sofrerem discriminação, de ter uma habitação, acesso à reabilitação, moradia, assistência social, transporte, mobilidade e acessibilidade (TAVARES; DUARTE; SENA, 2017). As crianças e os adolescentes com 
necessidades especiais devem ter sua individualidade reconhecida, para sentir-se sujeito no mundo (TENÓRIO, 2019).

Quadro 1 - Artigos que constituíram o corpus de análise.

\begin{tabular}{|c|c|c|c|c|}
\hline $\begin{array}{l}\text { Código de } \\
\text { identificação }\end{array}$ & Título & $\begin{array}{c}\text { Ano de } \\
\text { Publicação }\end{array}$ & Periódico & $\begin{array}{c}\text { Base de } \\
\text { Dados }\end{array}$ \\
\hline A1 & $\begin{array}{l}\text { Sobrecarga dos cuidadores de crianças ou adolescentes que } \\
\text { sofrem transtorno mental no município de Maringá - Paraná }\end{array}$ & 2016 & Escola Anna Nery & Scielo \\
\hline A2 & $\begin{array}{l}\text { Autocuidado apoiado para crianças e adolescentes com } \\
\text { doenças crônicas e suas famílias }\end{array}$ & 2017 & $\begin{array}{l}\text { Revista Brasileira de } \\
\text { Enfermagem }\end{array}$ & Scielo \\
\hline A3 & $\begin{array}{l}\text { Contextos de cuidado à criança/adolescente com Diabetes } \\
\text { Mellitus: uma abordagem socioambiental }\end{array}$ & 2018 & Aquichan & Scielo \\
\hline A4 & $\begin{array}{l}\text { Mãe-cuidadora de criança/adolescente com Paralisia } \\
\text { Cerebral: O cuidar de si }\end{array}$ & 2018 & Enfermería Global & Scielo \\
\hline A5 & $\begin{array}{l}\text { Tomando decisões na atenção à saúde de crianças/ } \\
\text { adolescentes com condições crônicas complexas: } \\
\text { uma revisão da literatura }\end{array}$ & 2019 & $\begin{array}{l}\text { Ciência \& Saúde } \\
\text { Coletiva }\end{array}$ & Scielo \\
\hline A6 & $\begin{array}{l}\text { Características pré e pós-natal de crianças e } \\
\text { adolescentes com deficiência intelectual }\end{array}$ & 2019 & Revista CEFAC & Lilacs \\
\hline A7 & $\begin{array}{l}\text { Ações de autocuidado apoiadas para crianças e } \\
\text { adolescentes com doenças crônicas }\end{array}$ & 2019 & $\begin{array}{l}\text { Texto \& Contexto - } \\
\text { Enfermagem }\end{array}$ & Scielo \\
\hline A8 & $\begin{array}{l}\text { Interação entre a família e a criança/adolescente com } \\
\text { deficiência auditiva }\end{array}$ & 2020 & $\begin{array}{l}\text { CoDAS (Distúrbios da } \\
\text { Comunicação, } \\
\text { Audiologia e deglutição) }\end{array}$ & Scielo \\
\hline A9 & $\begin{array}{l}\text { Uma existência solícita: o cuidado da mãe à criança/ } \\
\text { adolescente com paralisia cerebral }\end{array}$ & 2020 & $\begin{array}{l}\text { Bioscience journal } \\
\text { (Online) }\end{array}$ & Lilacs \\
\hline
\end{tabular}

Quadro 1: Caracterização das produções científicas na Scielo e Lilacs, busca realizada em 2020.

No que se refere ao cuidado de CRIANES, esse é apresentado como uma estratégia para a integração de várias esferas da vida, que incluam o desenvolvimento saudável. Elas contemplam os aspectos físico, emocional e espiritual para o crescimento e desenvolvimento (A9). Quanto aos locais em que os cuidados de crianças e adolescentes são realizados, percebe-se que as ações de cuidado são isoladas, em espaços distintos, seja nos espaços de saúde ou em espaços sociais, o que sugere a necessidade de ações em rede, que contemplem a criança e o adolescente em diferentes cenários de atenção (A5, A7).

Devido à continuidade do cuidado que a doença crônica exige, é comum que algumas CRIANES necessitem de hospitalizações prolongadas, o que pode gerar um aumento na demanda de cuidados. $\mathrm{Na}$ hospitalização, o cuidado é exercido pelos profissionais da equipe multidisciplinar, entre esses profissionais destacam-se a equipe de enfermagem. Já no domicílio, quem realiza os cuidados primários de CRIANES são os familiares/cuidadores. Por isso, é de extrema importância atender as necessidades de educação em saúde para o cuidado de CRIANES, a partir da realidade dos cuidadores familiares (SIMONASSE; MORAES, 2015).

O apoio dos profissionais de saúde para o cuidado possibilita melhorar a qualidade de vida e o progresso das condições de saúde de quem está sendo cuidado (FERNANDES et al., 2019). Portanto, os profissionais de saúde devem auxiliar no planejamento do cuidado e oferecer suporte 
às famílias cuidadoras que assumem o cuidado, e passam por situações singulares e complexas (THOMAZ et al., 2020)

Considera-se ainda, que as CRIANES podem ser cuidadas em diferentes contextos que envolvem tanto o domicílio, quanto a escola, a comunidade e os serviços de saúde. Para proporcionar esse cuidado é fundamental a constituição do vínculo, para que a criança e o adolescente tenham uma rede de apoio (A3). Destaca-se ainda, significativas mudanças no perfil do cuidado e da assistência pediátrica, onde o cuidado às crianças e aos adolescentes está apoiada nos serviços de saúde, com ênfase nos hospitais e ambulatórios (A5). Assim, é relevante a inserção de estratégias de apoio ao autocuidado dessa população e de suas famílias no âmbito da saúde pública (FERNANDES et al., 2017).

O ambiente domiciliar é o primeiro espaço de convivência onde são construídas as referências de vida, os valores familiares e a construção social. As crianças e os adolescentes que vivem com necessidades especiais precisam de um cuidado que extrapole a dimensão hospitalar, estendendo-se também ao domicílio (OLIVEIRA et al., 2018). O processo de cuidado de uma CRIANES deve ser apoiado em protocolos de cuidado, a fim de que os cuidados singulares e específicos as demandas de saúde possam ser supervisionados (A2). No que concerne a atenção primária de saúde, é necessário que os profissionais de saúde estejam preparados para acolher essas crianças, adolescentes e seus familiares com o intuito de constituir uma rede de apoio.

O apoio à família cuidadora de CRIANES é fundamental para a manutenção da vida, bem como o incentivo para a autonomia e o processo de autocuidado desses indivíduos (A7). As CRIANES são definidas como aquelas que necessitam de cuidados contínuos, especiais, temporários ou permanentes, por vezes complexos, para a manutenção da vida. Elas possuem uma variedade de cuidados singulares ainda maiores, quando comparadas às crianças e aos adolescentes que não apresentam necessidades especiais de saúde. As demandas vão além dos cuidados básicos exigidos no cotidiano diário, pois requerem de seus familiares diferentes formas de cuidar no espaço domiciliar após a alta hospitalar (VIANA et al., 2018).

O cuidado de crianças e adolescentes que vivem com demandas de cuidados de saúde podem causar repercussões no sistema familiar (A3). A sobrecarga de quem cuida diariamente de uma criança ou adolescente que apresenta necessidades especiais é intensa, visto que o cuidado está relacionado ao uso de medicação e acompanhamento da criança/adolescente nas atividades do cotidiano (A1). Essa sobrecarga dos cuidadores tem raízes na essência do cuidado para a manutenção da vida, em que o cuidador familiar sente obrigação em desenvolver o cuidado (A3). Quando as demandas de cuidados vêm acompanhadas por transtornos mentais na infância e adolescência, causa um desgaste físico e emocional ainda maiores nos familiares cuidadores (A1).

O processo de cuidado de CRIANES está condicionado ao cuidado cotidiano, presente em tarefas comuns como auxílio para a alimentação, ajuda para vestir, oferta de medicamentos, entre outras possibilidades. A necessidade especial de saúde acarreta transformações comportamentais do 
núcleo familiar que precisa fazer adaptações em prol da manutenção do cuidado, o que pode repercutir em outras esferas da vida, principalmente por não haver uma determinação temporal de melhora, prognóstico incerto e de longa ou indefinida duração. Além disso, essas crianças e adolescentes vivenciam o processo de hospitalizações recorrentes, e demandam um processo de cuidado contínuo (BURIOLA et al., 2016).

A respeito das estratégias para minimizar os impactos na prática de cuidado de CRIANES é apoiar a gestão do autocuidado de crianças e adolescentes, por meio do suporte profissional que contemple a criança, o adolescente e a família cuidadora (A2). Outra estratégia é a possibilidade do exercício da autonomia, em que os familiares cuidadores também advoguem em prol da autonomia das crianças e adolescentes, incentivando o cuidado e reconhecendo suas habilidades (A8). Os profissionais de saúde devem concentrar suas iniciativas em prol da autonomia e cuidado desses indivíduos (A6).

As estratégias de cuidado de crianças e adolescentes que vivenciam situações crônicas de saúde, requer um cuidado integral, organizado, contínuo e coordenado por profissionais de saúde (SILVA et al., 2018). A condição crônica na infância apresenta inúmeras consequências e repercussões diante da doença, do contexto social, dos laços estabelecidos, que emergem a participação da criança, do adolescente e da família no cuidado (REIS et al., 2017). No cotidiano, cabe à família realizar o papel de preparação do adolescente com necessidades especiais de saúde para o futuro, ensinando-os a conviver com as limitações impostas pela doença e incentivando-os na realização de atividades que melhorem sua independência na vida adulta (RAMOS et al., 2018).

Quanto ao exercício do cuidado às CRIANES está atrelado às mulheres da família (A4). Algumas mães de CRIANES acreditam que fazem parte da corporeidade do filho, como se a criança fosse uma extensão delas (A9). Esse dado vai ao encontro de um estudo desenvolvido por Freitag et al. (2020), em que o vínculo entre mãe e filho é intenso ao ponto de a mãe ser compreendida como parte da corporeidade do filho. Essas mulheres mães abdicam de suas vidas pessoais, desenvolvem cuidados complexos e cuidam de forma solitária de seus filhos (A4).

Observa-se ainda, relações de cuidado em que as mulheres historicamente assumiram o protagonismo do cuidado vinculado a papeis de gênero, cuja função de cuidar é atribuída às mulheres da família e aos homens a função de provedores do sustento familiar (DALTRO; MORAES; MARSIGLIA, 2018). Os cuidados domiciliares de CRIANES está ancorado em uma sociedade com divisão de tarefas, sendo na maioria das vezes, designada à mulher a missão de gerar e promover o cuidado para seu filho (RAMOS et al., 2017). A diversidade de cuidados exigidos pelas CRIANES reduz a oportunidade das mães e da família na participação de atividades sociais, de lazer, e, também, no estabelecimento e manutenção de novos laços de amizade (SIMONASSE; MORAES, 2015).

Neste contexto, é primordial a participação de outros membros da família no processo de cuidado em prol do desenvolvimento da CRIANES. A inclusão paterna nas tarefas de cuidado são fundamentais, pois além de evitar a sobrecarga da mãe, também promove o vínculo e a participação 
no cotidiano da CRIANES (DALTRO, MORAES; MARSIGLIA, 2018). A inclusão do pai ou de outros membros da família no cuidado possibilita partilhar as responsabilidades, promover a interação social e a convivência que são fundamentais para a afetividade, a comunicação, a interatividade e a socialização da CRIANES.

No que se refere a interação da CRIANES com a família, é essencial considerar as potencialidades e as fragilidades, sobretudo de comunicação nos casos de criança/adolescente com deficiência auditiva (A8). Para os familiares de CRIANES que apresentam doenças mentais, os impactos são extensos porque requer que os cuidadores/familiares coloquem as suas necessidades e desejos em segundo plano, e modifiquem seu cotidiano de vida (A1). Desta forma, cabe aos profissionais de saúde conhecer acerca dessa temática, a fim de intervir em diferentes espaços que possam ser prósperos para o cuidado e saúde de crianças e adolescentes que vivem com algum tipo de deficiência (A3, A5, A6).

Quando se trata da rotina de cuidado, faz-se necessário que pais e mães estejam trabalhando em conjunto na prestação de cuidados às crianças/adolescentes com doença crônica a fim de reduzir a sobrecarga provocada pelo gerenciamento do cuidado (RAMOS et al., 2017; DALTRO, MORAES; MARSIGLIA, 2018). A sobrecarga de cuidado faz com que os familiares não tenham tempo para o cuidado de si e muitas vezes, os levam a vivenciar o isolamento social (FREITAG; MILBRATH; MOTA, 2018). Assim, é importante um olhar atento à saúde de crianças e adolescentes, extensivas aos familiares cuidadores que estão participando de forma ativa no processo de cuidado e manutenção da saúde das CRIANES.

O impacto da necessidade especial acaba atingindo vários aspectos da vida familiar, requer que os cuidadores deixem suas necessidades e coloquem as da criança/adolescente em primeiro lugar. Portanto, a família necessita de apoio e cuidado por parte das redes de atenção à saúde (BURIOLA et al., 2016). Desta forma, o vínculo entre a rede de apoio e a família, repercute positivamente no cuidado desenvolvido pelos familiares cuidadores de crianças e adolescentes que vivem com necessidades especiais de saúde (SILVA et al., 2017; QUINTANILHA et al., 2017).

\section{CONSIDERAÇÕES FINAIS}

O cuidado de CRIANES perpassa o cuidado materno, familiar e ao processo de autocuidado de familiares cuidadores que assumem o protagonismo do cuidado de CRIANES. De modo geral, o cuidado é exercido pelas mães de CRIANES que abnegam de seus anseios pessoais em prol do cuidado exclusivo de seus filhos. Este aspecto pode gerar uma sobrecarga materna, por serem cuidadoras exclusivas de seus filhos.

Os achados revelam sobre a necessidade de reconhecer as demandas de cuidados, e as condições de saúde das CRIANES revelam a importância de constituir uma rede articulada de profissionais e serviços de saúde, que possam atender as diferentes demandas de cuidados dessa clientela. 
Cada criança e adolescente que apresenta demandas de cuidados de saúde tem uma história singular, que requer atenção especial para a manutenção da vida. Além disso, os serviços de saúde devem estar preparados para acolher os familiares cuidadores, visto que desempenham um papel fundamental no exercício do cuidado, mesmo que não tenham formação específica para isso. As famílias vão articulando diferentes maneiras de cuidar, a partir de suas vivências cotidianas no exercício do cuidado domiciliar de CRIANES.

Por fim, o estudo denota sobre a necessidade de uma rede de apoio social para a construção desse cuidado, aliado a rede de serviços de saúde. Os profissionais de saúde, entre eles os profissionais de enfermagem, são fundamentais no suporte aos familiares cuidadores de CRIANES.

Quanto às limitações do estudo, tem-se a escassa quantidade de publicações nacionais referentes à essa temática. Neste sentido, sugere-se o desenvolvimento de novas pesquisas que ampliem as discussões acerca do cuidado domiciliar exercido pelos familiares cuidadores de CRIANES e as estratégias utilizadas para o cotidiano de cuidados.

\section{REFERÊNCIAS}

BRASIL. Ministério da Saúde. Lei no 8.069, de 13 de julho de 1990. Dispõe sobre o Estatuto da Criança e do Adolescente e dá outras providências - Brasília: 2009. Disponível em: https://bit.ly/33u4lme. Acesso em: dez. 2011.

BRASIL. Lei No 8.069, de 13 de julho de 1990. Dispõe sobre o Estatuto da Criança e do Adolescente e dá outras providências. Diário Oficial da República Federativa do Brasil, Brasília, DF, 16 jul. 1990. Disponível em: https://bit.ly/2R7CLbX. Acesso em: abr.2020.

BRASIL. Ministério da Saúde. Secretaria de Atenção à Saúde. Departamento de Ações Programáticas e Estratégicas. Proteger e cuidar da saúde de adolescentes na atenção básica. 1. ed. Brasília. 2017.

BURIOLA, A. A. et al. Sobrecarga dos cuidadores de crianças ou adolescentes que sofrem transtorno mental no município de Maringá - Paraná. Escola Anna Nery Revista de Enfermagem, v. 20, n. 2 , p. 344-351, 2016.

CABRAL, I. E. et al. A criança egressa da terapia intensiva na luta pela sobrevida. Revista Brasileira de Enfermagem, v. 57, n. 1, 35-9, 2004.

DALTRO, M. C. DE S. L.; MORAES, J. C. DE; MARSIGLIA, R. G. Cuidadores de crianças e adolescentes com transtornos mentais: mudanças na vida social, familiar e sexual. Saúde e Sociedade, v. 27, n.2, 544-555, 2018. 
FERNANDES, L. T. B. et al. Ações de autocuidado apoiado a crianças e adolescentes com doenças crônicas. Texto e Contexto Enfermagem, v. 28, e20180325, 2019.

FERNANDES, L. T. B. et al. Autocuidado apoiado para crianças e adolescentes com doença crônica e seus familiares. Revista Brasileira de Enfermagem, v. 70, n. 6, p. 1318-1329, 2017.

FIGUEIREDO, S. V.; SOUSA A. C. C.; GOMES I. L. V. Menores com necessidades especiais de saúde e familiares: implicações para a Enfermagem. Revista Brasileira de Enfermagem, v. 69, n. 1, p. 79-85, 2016.

FREITAG, V. L. et al. A willing existence: the care of the mother to the child. Bioscience Journal, v. 36, n. 1, 2020.

FREITAG, V. L; MILBRATH, V. M; MOTTA, M. DA G. C. DA. Madre-cuidadora de niño/adolescente con parálisis cerebral: El cuidado de sí misma. Enfermagem Global, v. 17, n. 50, p. 325-360, 2018.

INÁCIO, A. L. R.; PEIXOTO, A. P. G. L. A assistência de enfermagem e o cuidado familiar às crianças com necessidades especiais de saúde: uma revisão integrativa. Revista de Atenção à Saúde, v. 15, n. 53, p. 87-94, 2017.

LEITE, F. L. L. de M. et al. Criança com necessidades especiais de saúde: análise do cuidado prestado pela família. Revista Eletrônica Acervo Saúde, v. 11, n. 15, e1342, 2019.

MCPHERSON, M. G. et al. Uma nova definição de crianças com necessidades especiais de saúde. Pediatrics, v. 102, n. 1, p. 137-139, 1998.

NEVES E. T. et al. Rede de cuidados de crianças com necessidades especiais de saúde. Texto e Contexto Enfermagem, v. 24, n. 2, p. 399-406, 2015.

NÓBREGA, V. M. et al. Doença crônica na infância e adolescência: continuidade do cuidado na Rede de Atenção à Saúde. Revista Escola enfermagem USP, v. 51, e03226, 2017.

OLIVEIRA, S. M. de. et al. Contextos de cuidado à criança/adolescente com Diabetes Mellitus: uma abordagem socioambiental. Aquichan, v. 18, n. 1, p. 69-79, 2018. 
QUINTANILHA, B. C. et al. A produção do cuidado em um centro de atenção psicossocial infanto-juvenil. Mental, v. 11, n.20, p. 261-278, 2017.

RAMOS, R. M. et al. Cuidado paterno à criança e ao adolescente com doença crônica: percepção materna. Revista Gaúcha de Enfermagem, v. 38, n. 3, e0006, 2017.

REIS, K. M. N. et al. A vivência da família no cuidado domiciliar à criança com necessidades especiais de saúde. Ciência Enfermagem, v. 23, n. 1, p. 45-55, 2017.

ROTHER, E. T. Revisão sistemática X revisão narrativa. Acta Paulista Enfermagem, v. 20, n. 2, p. V-VI, 2007.

SILVA, R. M. M. DA. et al. A longitudinalidade no cuidado à saúde da criança no contexto da atenção primária. Revista Enfermagem UFPE, v. 11, n. 5, p. 1909-1917, 2017.

SILVA, M. E. DE A. et al. Doença crônica na infância e adolescência: vínculos da família na rede de atenção à saúde. Texto e Contexto Enfermagem, v.27, n. 2, e4460016, 2018.

SILVEIRA, A. DA.; NEVES, E. T. Dimensão política do cuidado às Crianças e Adolescentes com Necessidades Especiais de Saúde: uma reflexão. Revista Enfermagem UFSM, v. 7, n. 2, p. 337-346, 2017.

SIMONASSE, M. F.; MORAES, J. R. M. M. DE. Crianças com necessidades especiais de saúde: impacto no cotidiano familiar. Revista de Pesquisa Cuidado é Fundamental, v. 7, n. 3, p. 2902-2909, 2015.

TAVARES, T. S.; DUARTE, E. D.; SENA, R. R. DE. Direitos sociais das crianças com condições crônicas: análise crítica das políticas públicas brasileiras. Escola Anna Nery Revista de Enfermagem, v. 21, n. 4, e20160382, 2017.

TENÓRIO, J. C. S. Adolescência e Vida Contemporânea: uma Relação entre Limite e Identidade. Psicologado. 8. ed. 2015.

THOMAZ, M. M. et al. Interação entre a família e a criança/adolescente com deficiência auditiva. CoDAS, v. 32, n. 6, e20190147, 2020.

VIANA, I. DA S. et al. Encontro educativo da enfermagem e da família de crianças com necessidades especiais de saúde. Texto e Contexto Enfermagem, v. 27, n. 3, e5720016, 2018. 
